\title{
Decoupling of modes for the elastic wave equation in media of limited smoothness
}

\author{
Valeriy Brytik ${ }^{1} \quad$ Maarten V. de Hoop ${ }^{1} \quad$ Hart F. Smith ${ }^{2} \quad$ Gunther Uhlmann ${ }^{2}$
}

November 14, 2010

${ }^{1}$ Center for Computational and Applied Mathemematics, and Geo-Mathematical Imaging Group, Purdue University, West Lafayette, Indiana, USA.

${ }^{2}$ Department of Mathematics, University of Washington, Seattle, Washington, USA.

\begin{abstract}
We establish a decoupling result for the $P$ and $S$ waves of linear, isotropic elasticity, in the setting of twicedifferentiable Lamé parameters. Precisely, we show that the $P \leftrightarrow S$ components of the wave propagation operator are regularizing of order one on $L^{2}$ data, by establishing the diagonalization of the elastic system modulo a $L^{2}$-bounded operator. Effecting the diagonalization in the setting of twice-differentiable coefficients depends upon the symbol of the conjugation operator having a particular structure.
\end{abstract}

\section{Introduction}

We consider the linear, isotropic elastic wave equation on $\mathbb{R}^{n}$, and Cauchy initial value problem on a time interval $\left[-t_{0}, t_{0}\right]$,

$$
\left\{\begin{aligned}
\partial_{t}^{2} w_{i}-(A(x, D) w)_{i} & =0, \\
\left.w_{i}\right|_{t=0} & =f_{i}, \\
\left.\partial_{t} w_{i}\right|_{t=0} & =g_{i},
\end{aligned}\right.
$$

where $w=\left(w_{1}, \ldots, w_{n}\right)$ denotes the displacement vector, and

$$
(A(x, D) w)_{i}=\sum_{k=1}^{n} \partial_{k}\left(\mu(x)\left(\partial_{k} w_{i}+\partial_{i} w_{k}\right)\right)+\partial_{i}\left(\lambda(x) \partial_{k} w_{k}\right) .
$$

The elastic wave equation arises in many physical situations, including the propagation of seismic waves through the Earth. The identification and separation of $P$ and $S$ polarized constituents (so-called phases) underlies many applications of seismic data processing and imaging, which traditionally has been based on the assumption of smoothness of the medium and asymptotic considerations. As an example, we mention the notion of receiver functions, which were introduced and developed by Vinnik (1977) and Langston (1979).

Throughout this paper, we assume that the Lamé parameter functions $\mu$ and $\lambda$ belong to $C^{1,1}\left(\mathbb{R}^{n}\right)$, in that the first derivatives $\nabla \mu$ and $\nabla \lambda$ are globally bounded, Lipschitz functions on $\mathbb{R}^{n}$.

The principal symbol $\sigma_{2}(A)(x, \xi)$ of $A(x, D)$ takes the form

$$
\begin{aligned}
-\sigma_{2}(A) & =\mu(x)|\xi|^{2} \mathrm{I}+(\mu(x)+\lambda(x)) \xi \otimes \xi \\
& =(2 \mu(x)+\lambda(x))|\xi|^{2} \sigma_{0}\left(\Pi_{P}\right)+\mu(x)|\xi|^{2} \sigma_{0}\left(\Pi_{S}\right)
\end{aligned}
$$


where $\Pi_{P}$ and $\Pi_{S}$ are the projection operators

$$
\Pi_{P} w=\Delta^{-1} \nabla(\operatorname{div} w), \quad \Pi_{S} w=\left(\mathrm{I}-\Pi_{P}\right) w
$$

corresponding to the order 0 homogeneous Fourier multipliers

$$
\sigma_{0}\left(\Pi_{P}\right)_{i l}=\frac{\xi_{i} \xi_{l}}{|\xi|^{2}}, \quad \sigma_{0}\left(\Pi_{S}\right)_{i l}=\frac{|\xi|^{2} \delta_{i l}-\xi_{i} \xi_{l}}{|\xi|^{2}} .
$$

$\Pi_{P}$ and $\Pi_{S}$ are thus homogeneous Calderón convolution operators. The symbols of $\Pi_{P}$ and $\Pi_{S}$ are, for each $x$ and $\xi$, projections onto the eigenspaces of $A(x, \xi)$, respectively of dimension 1 and $n-1$. This yields that

$$
\begin{aligned}
& A(x, D) \Pi_{P} w=(2 \mu(x)+\lambda(x)) \Delta \Pi_{P} w+\text { l.o.t. } \\
& A(x, D) \Pi_{S} w=\mu(x) \Delta \Pi_{S} w+\text { l.o.t., }
\end{aligned}
$$

where l.o.t. denotes an operator of first order in $w$ (on some finite range of Sobolev spaces, as discussed in the next section.)

We call $\Pi_{P} w$ and $\Pi_{S} w$, respectively, the $P$ and $S$ modes of the elastic wave $w$. If the Lamé parameters are constant, then the wave propagation operator commutes with $\Pi_{P}$ and $\Pi_{S}$, so that wave evolution preserves modes which are of pure $P$ or pure $S$ form.

In the case of nonconstant $\mu$ and $\lambda$, there will in general be a nonvanishing coupling of the $P$ and $S$ modes in the evolution. The main result of this paper is that the $P \leftrightarrow S$ terms in the wave propagator are one order smoother than the $P \rightarrow P$ and $S \rightarrow S$ terms. Precisely, let the solution to (1.1) be written in the form

$$
w(t, \cdot)=\mathbf{c}(t) f+\mathbf{s}(t) g,
$$

and decompose $\mathbf{c}(t)=\mathbf{c}_{P P}(t)+\mathbf{c}_{P S}(t)+\mathbf{c}_{S P}(t)+\mathbf{c}_{S S}(t)$, where $\mathbf{c}_{P P}(t)=\Pi_{P} \mathbf{c}(t) \Pi_{P}$, etc. We similarly decompose $\mathbf{s}(t)$. The operators $\mathbf{c}_{P P}(t)$ and $\mathbf{c}_{S S}(t)$ are bounded on a range of Sobolev spaces $H^{s}\left(\mathbb{R}^{n}\right)$, in particular for $0 \leq s \leq 1$, and $\mathbf{s}_{P P}(t), \mathbf{s}_{S S}(t)$ map $H^{s-1}\left(\mathbb{R}^{n}\right)$ to $H^{s}\left(\mathbb{R}^{n}\right)$ for the same range. (All maps are strongly continuous in $t$.) We prove that

$$
\mathbf{c}_{P S}(t): L^{2}\left(\mathbb{R}^{n}\right) \rightarrow H^{1}\left(\mathbb{R}^{n}\right), \quad \mathbf{s}_{P S}(t): H^{-1}\left(\mathbb{R}^{n}\right) \rightarrow H^{1}\left(\mathbb{R}^{n}\right) .
$$

Additionally, we show that $\mathbf{c}_{P P}(t)$ agrees with the propagator for $A_{P P}=\Pi_{P} A \Pi_{P}$ up to a similarly regularizing term; see Corollary 3.3 for a precise statement.

Establishing regularization for the $P \leftrightarrow S$ components of the propagator requires diagonalizing $A(x, D)$ to second order; that is, one order beyond the principal symbol consideration of (1.4), since treating a first order derivative of $w$ as a driving force term will produce terms of the same order as $w$ in the solution. This diagonalization is carried out through conjugation by $I+K$, where $K$ is a compound pseudodifferential operator with finite regularity symbol of order -1 .

In the case of smooth $\mu$ and $\lambda$, a similar diagonalization was carried out, in a more general setting, to all orders in Taylor (1975) using the pseudodifferential calculus. He used this diagonalization to study the reflection of singularities for the system of isotropic elasticity, as well as for other systems with constant multiplicities. Stolk and de Hoop (2002) have used Taylor's diagonalization in the study of the linearized seismic inverse scattering problem. Dencker (1982) analyzed the propagation of polarization in Euclidean space for linear systems of principal type with smooth coefficients, which includes isotropic elasticity. Wang (1998) applied Dencker's propagation of polarization result to the linearized seismic inverse problem; in particular he discusses the issue of polarization in reflections caused by discontinuities of a perturbation of the medium.

In the case of $C^{1,1}$ coefficients, the construction of $K$ is more delicate, and carrying through the composition calculus to first order depends on the symbol of $K$ having a special form. We believe that this is the first article studying the interaction of $P$ and $S$ waves for the elastic system in non-smooth media, and it remains open under what conditions this can be carried out in the $C^{1,1}$ setting for more general diagonalizable 
systems with constant multiplicity eigenvalues. We anticipate that our result will have applications in the study of the linearized seismic inverse problem in media of limited smoothness. There has been considerable activity in studying unique continuation in the stationary case for non-smooth Lamé parameters, see Lin et. al. (2010) and the references therein for the most recent results.

\section{Notation.}

All pseudodifferential operators that arise in this paper will have symbols that are finite sums $p(x, \xi)=$ $\sum_{j=0}^{N} p_{m-j}(x, \xi)$, where $p_{m-j}$ is homogeneous in $\xi$ of degree $m-j$. We use $\sigma_{m}(p)$ to denote the principal symbol $p_{m}(x, \xi)$ of $p$, where $m$ is the order of $p$.

We will use $L^{2}\left(\mathbb{R}^{n}\right)$ and $H^{s}\left(\mathbb{R}^{n}\right)$ to denote the $L^{2}$ and $L^{2}$-based Sobolev spaces, in the case of complex valued functions as well as $n$-dimensional vector valued functions, such as the solution $w$.

Throughout the paper, $R$ will denote an operator that is bounded on $L^{2}\left(\mathbb{R}^{n}\right)$; the form of $R$ may change in each occurrence.

\section{The Conjugation Operator}

In this section we construct the conjugation operator $K$, which diagonalizes $A(x, D)$ up to a $L^{2}$-bounded operator. We decompose $A=A_{P P}+A_{P S}+A_{S P}+A_{S S}$, where

$$
\begin{aligned}
& A_{P P}=\Pi_{P} A(x, D) \Pi_{P}, \quad A_{P S}=\Pi_{P} A(x, D) \Pi_{S}, \\
& A_{S P}=\Pi_{S} A(x, D) \Pi_{P}, \quad A_{S S}=\Pi_{S} A(x, D) \Pi_{S} .
\end{aligned}
$$

Note that $A_{S P}=A_{P S}^{*}$, while both $A_{S S}$ and $A_{P P}$ are self-adjoint. Furthermore $A_{S P}$, and hence $A_{P S}$, is of order 1 , in that

$$
A_{P S}: H^{s+1}\left(\mathbb{R}^{n}\right) \rightarrow H^{s}\left(\mathbb{R}^{n}\right) \text { for } \quad-2 \leq s \leq 1
$$

To see this, we write

$$
A_{P S} w=\Delta^{-1} \nabla\left(\operatorname{div} A \Pi_{S} w\right)=\Delta^{-1} \nabla\left([\operatorname{div}, A] \Pi_{S} w\right),
$$

where we use $\operatorname{div} \Pi_{S} \equiv 0$. Each component of the composition then takes the form $\Delta^{-1} \nabla\left(\partial\left(b \partial \Pi_{S} w\right)\right)$, where $b$ denotes a first-order derivative of $\mu$ or $\lambda$, which being Lipschitz acts as a multiplier on $H^{s}$ for $-1 \leq s \leq 1$. This establishes (2.1) for such $s$. If instead one writes

$$
A_{P S} w=-\Pi_{P} A(\Delta-\nabla \operatorname{div}) \Delta^{-1} w
$$

and commutes one derivative from the right past $A$, one obtains (2.1) for the range $-2 \leq s \leq 0$.

Our main conjugation result is the following.

Lemma 2.1 There exists an operator $K$ of order -1 , in the sense that

$$
K: H^{s}\left(\mathbb{R}^{n}\right) \rightarrow H^{s+1}\left(\mathbb{R}^{n}\right) \text { for } \quad-1 \leq s \leq 1,
$$

such that

$$
\begin{aligned}
& \Pi_{P}(I+K) A(x, D) w=A_{P P}(I+K) w+R_{P} w \\
& \Pi_{S}(I+K) A(x, D) w=A_{S S}(I+K) w+R_{S} w
\end{aligned}
$$

where $R_{P}$ and $R_{S}$ are bounded maps on $L^{2}\left(\mathbb{R}^{n}\right)$.

Additionally,

$$
K_{P P}=K_{S S}=0, \quad K_{S P}^{*}=-K_{P S},
$$

where $K_{P P}=\Pi_{P} K \Pi_{P}, K_{S P}=\Pi_{S} K \Pi_{P}$, etc. 
To establish (2.4)-(2.5), we expand $A$ and $K$ in terms of their $P$ and $S$ components. Terms such as $\Pi_{P} K_{S P}$ and $A_{P P} K_{S P}$ vanish. By (2.1) and (2.3), the terms $K_{P S} A_{S P}$ and $K_{S P} A_{P S}$ are bounded on $L^{2}\left(\mathbb{R}^{n}\right)$, hence are absorbed into $R$. Using (2.6), the conditions (2.4)-(2.5) thus reduce to the following conditions

$$
\begin{aligned}
& K_{P S} A_{S S}-A_{P P} K_{P S}+A_{P S}=R, \\
& K_{S P} A_{P P}-A_{S S} K_{S P}+A_{S P}=R,
\end{aligned}
$$

meaning precisely that the left hand sides are bounded maps on $L^{2}\left(\mathbb{R}^{n}\right)$. Condition (2.8) is equivalent to (2.7) by taking adjoints, so we will establish (2.7).

Consider first the case of smooth $\mu$ and $\lambda$. Since $K_{P S}$ is of order -1 , and $A_{P P}$ and $A_{S S}$ of order 2, the equality (2.7) reduces to saying that the top (i.e. first) order terms in the symbol expansion for the left hand side cancel out. By (1.3), this implies that

$$
\sigma_{-1}\left(K_{P S}\right)=\frac{-\sigma_{1}\left(A_{P S}\right)}{(\mu(x)+\lambda(x))|\xi|^{2}} .
$$

We will also reduce (2.7) to a principal symbol calculation for the case of $\mu$ and $\lambda$ of regularity $C^{1,1}$. Care needs to be taken, however, since by (2.2) the symbol of $A_{P S}$, hence $K_{P S}$, is only Lipschitz in $x$, whereas $A_{P P}$ and $A_{S S}$ are of second order. That we can reduce matters to a symbol calculation depends critically on the fact that $K_{P S}$ has a particular structure, which in turn depends on the special form of $A_{P S}$.

We consider the operator $A_{P S}$ more closely. Since div $\Pi_{S}=0$, by (1.2) we can write

$$
\begin{aligned}
\left(A_{P S} w\right)_{i} & =\sum_{j, k=1}^{n} \Delta^{-1} \partial_{i} \partial_{j} \partial_{k}\left(\mu\left(\partial_{k}\left(\Pi_{S} w\right)_{j}+\partial_{j}\left(\Pi_{S} w\right)_{k}\right)\right) \\
& =2 \sum_{j, k=1}^{n} \Delta^{-1} \partial_{i} \partial_{j}\left(\left(\partial_{k} \mu\right) \partial_{j}\left(\Pi_{S} w\right)_{k}\right) \\
& =2 \sum_{k=1}^{n} \partial_{i}\left(\left(\partial_{k} \mu\right)\left(\Pi_{S} w\right)_{k}\right)-2 \sum_{j, k=1}^{n} \Delta^{-1} \partial_{i} \partial_{j}\left(\left(\partial_{j} \partial_{k} \mu\right)\left(\Pi_{S} w\right)_{k}\right)
\end{aligned}
$$

In going from the first to the second line we used that $\sum_{k} \partial_{k}\left(\Pi_{S} w\right)_{k}=0$, together with symmetry of the expression in $j, k$. The second term in the third row is a bounded operator on $L^{2}$; hence we may write

$$
A_{P S} w=2 \nabla\left((\nabla \mu) \cdot \Pi_{S} w\right)+R .
$$

Motivated by (2.9), we now define

$$
K_{P S} w=2 \Phi(D) \Delta^{-1} \nabla\left((\mu+\lambda)^{-1}(\nabla \mu) \cdot \Pi_{S} w\right),
$$

and set $K_{S P}=-K_{P S}^{*}$ so that (2.6) holds. Here, $\Phi(\xi)$ is a smooth cutoff to $|\xi| \geq M$,

$$
\Phi(\xi)= \begin{cases}1, & |\xi| \geq 2 M, \\ 0, & |\xi| \leq M,\end{cases}
$$

which we introduce into the definition to avoid singularities of the negative order multiplier $\Delta^{-1} \nabla$ at $\xi=0$. We will choose $M$ depending on the $C^{1,1}$ norms of $\mu$ and $\lambda$. Condition (2.3) holds since $(\lambda+\mu)^{-1} \nabla \mu$ is a multiplier on $H^{s}$ for $-1 \leq s \leq 1$. Furthermore, by taking $M$ large, we will have

$$
\|K w\|_{H^{s}} \leq \frac{1}{2}\|w\|_{H^{s}}, \quad-1 \leq s \leq 1 .
$$

Finally, we note that since $K_{P S} w$ is the gradient of a function, it follows that

$$
K_{P S}=\Pi_{P} K_{P S}=K_{P S} \Pi_{S} .
$$


To evaluate the composition $A_{P P} K_{P S}$, we observe that we may write

$$
A_{P P}=(2 \mu+\lambda) \Delta \Pi_{P}+\sum_{k=1}^{n} R_{k} \partial_{k}
$$

where

$$
R_{k}: H^{s}\left(\mathbb{R}^{n}\right) \rightarrow H^{s}\left(\mathbb{R}^{n}\right), \quad-1 \leq s \leq 1 .
$$

This follows from the fact that the commutator $\left[\Pi_{P} \partial_{k}, \mu\right]$ is bounded on $H^{s}\left(\mathbb{R}^{n}\right)$ over this range, which is a consequence of the Calderón commutator theorem; see Calderón (1965). Indeed, $\Pi_{P} \partial_{k}$ is a first order homogenous multiplier, yielding boundedness on $L^{2}$ by Calderón's theorem. Then $\left[\nabla,\left[\Pi_{P} \partial_{k}, \mu\right]\right]=\left[\Pi_{P} \partial_{k}, \nabla \mu\right]$ is similarly bounded on $L^{2}$, since $\nabla \mu$ is Lipschitz, yielding the result.

Since $\partial_{k} \circ K_{P S}$ is bounded on $L^{2}$, it follows that

$$
\begin{aligned}
A_{P P} K_{P S} w & =2(2 \mu+\lambda) \Phi(D) \nabla\left((\mu+\lambda)^{-1}(\nabla \mu) \cdot \Pi_{S} w\right)+R w \\
& =2 \Phi(D) \nabla\left((2 \mu+\lambda)(\mu+\lambda)^{-1}(\nabla \mu) \cdot \Pi_{S} w\right)+R w .
\end{aligned}
$$

To evaluate the composition $K_{P S} A_{S S}$, we first observe that we can write

$$
A_{S S} w=\Pi_{S} \Delta\left(\mu \Pi_{S} w\right)+A_{1} w,
$$

where $A_{1}: L^{2} \rightarrow H^{-1}$. To see this, we note that $\operatorname{div} \Pi_{S} \equiv 0$, so the $\lambda$ terms in $A$ do not contribute to $A_{S S}$. For the same reason we can write

$$
\Pi_{S} \sum_{k} \partial_{k}\left(\mu \partial_{i}\left(\Pi_{S} w\right)_{k}\right)=\Pi_{S} \sum_{k}\left(\partial_{k} \mu\right) \partial_{i}\left(\Pi_{S} w\right)_{k}
$$

which maps $L^{2} \rightarrow H^{-1}$. Finally, we write

$$
\Pi_{S} \sum_{k} \partial_{k}\left(\mu \partial_{k}\left(\Pi_{S} w\right)_{i}\right)=\Pi_{S} \Delta\left(\mu \Pi_{S} w\right)_{i}-\Pi_{S} \sum_{k}\left(\partial_{k} \mu\right) \partial_{k}\left(\Pi_{S} w\right)_{i},
$$

and observe that the last term maps $L^{2} \rightarrow H^{-1}$.

By (2.3), the composition $K_{P S} A_{1}$ is bounded on $L^{2}$, hence we are reduced to showing that

$$
\Phi(D) \Delta^{-1} \nabla\left((\mu+\lambda)^{-1}(\nabla \mu) \cdot(\Delta-\nabla \operatorname{div})\left(\mu \Pi_{S} w\right)\right)=\Phi(D) \nabla\left((\mu+\lambda)^{-1}(\nabla \mu) \cdot\left(\mu \Pi_{S} w\right)\right)+R w,
$$

where we write $\Pi_{S} \Delta=\Delta-\nabla$ div, and understand that $\Delta$ acts on each component of a vector, i.e. as a scalar operator.

The relation (2.12) is derived as a consequence of the identities

$$
\begin{aligned}
\Delta(V \cdot W) & =V \cdot \Delta W+2 \operatorname{div}(W \cdot \nabla V)-W \cdot(\Delta V) \\
V \cdot \nabla \operatorname{div} W & =\operatorname{div}(V \operatorname{div} W)-\operatorname{div}(W \operatorname{div} V)+W \cdot \nabla \operatorname{div} V
\end{aligned}
$$

where in the term $\operatorname{div}(W \cdot \nabla V)$ the div pairs with $\nabla$.

Setting $V=(\mu+\lambda)^{-1} \nabla \mu$, and $W=\mu \Pi_{S} w$, then (2.12) is equivalent to

$$
\Phi(D) \Delta^{-1} \nabla((V \cdot(\Delta-\nabla \operatorname{div}) W-\Delta(V \cdot W))=R w .
$$

By (2.13)-(2.14) we can write the left hand side as

$$
\Phi(D) \Delta^{-1} \nabla(W \cdot(\Delta-\nabla \operatorname{div}) V-\operatorname{div}(V \operatorname{div} W)+\operatorname{div}(W \operatorname{div} V)-2 \operatorname{div}(W \cdot \nabla V)) .
$$


The last two terms,

$$
\Phi(D) \Delta^{-1} \nabla \operatorname{div}(W \operatorname{div} V-2 W \cdot \nabla V)
$$

are $L^{2}$ bounded operators applied to $W$, hence of the form $R w$, since $V$ is Lipschitz. Since $\operatorname{div} W=$ $(\nabla \mu) \cdot \Pi_{S} w$, the same holds for $\Phi(D) \Delta^{-1} \nabla \operatorname{div}(V \operatorname{div} W)$. The remaining term is where we use the special structure, since it involves two derivatives of $V$. The key observation is that

$$
(\Delta-\nabla \operatorname{div}) V=(\Delta-\nabla \operatorname{div})\left((\mu+\lambda)^{-1} \nabla \mu\right) \in L^{\infty}\left(\mathbb{R}^{n}\right),
$$

since $\Delta \nabla \mu=\nabla \operatorname{div} \nabla \mu$, and hence the expression in fact involves only second derivatives of $\mu$ and $\lambda$. Consequently,

$$
w \rightarrow \Phi(D) \Delta^{-1} \nabla(W \cdot(\Delta-\nabla \operatorname{div}) V): L^{2}\left(\mathbb{R}^{n}\right) \rightarrow H^{1}\left(\mathbb{R}^{n}\right) .
$$

\section{The Parametrix and Decoupling of Modes}

We will realize the parametrix construction for (1.1) using parametrices for the purely polarized equations, following the procedure introduced in Smith (1998).

Theorem 3.1 There exist strongly continuous one parameter families of operators

$$
\begin{array}{rlrl}
\mathbf{c}_{P}(t): H^{s}\left(\mathbb{R}^{n}\right) & \rightarrow H^{s}\left(\mathbb{R}^{n}\right), & \mathbf{s}_{P}(t): H^{s-1}\left(\mathbb{R}^{n}\right) & \rightarrow H^{s}\left(\mathbb{R}^{n}\right), \\
\partial_{t} \mathbf{c}_{P}(t): H^{s}\left(\mathbb{R}^{n}\right) & \rightarrow H^{s-1}\left(\mathbb{R}^{n}\right), & \partial_{t} \mathbf{s}_{P}(t): H^{s-1}\left(\mathbb{R}^{n}\right) \rightarrow H^{s-1}\left(\mathbb{R}^{n}\right),
\end{array}
$$

for $0 \leq s \leq 2$ and $t \in\left[-t_{0}, t_{0}\right]$, such that the solution to the Cauchy problem

$$
\left(\partial_{t}^{2}-A_{P P}\right) v_{P}=G_{P},\left.\quad v_{P}\right|_{t=0}=f_{P},\left.\quad \partial_{t} v_{P}\right|_{t=0}=g_{P},
$$

where $\Pi_{S} f_{P}=\Pi_{S} g_{P}=\Pi_{S} G_{P}=0$, and

$$
f_{P} \in H^{s}\left(\mathbb{R}^{n}\right), \quad g_{P} \in H^{s-1}\left(\mathbb{R}^{n}\right), \quad G_{P} \in L^{1}\left(\left[-t_{0}, t_{0}\right], H^{s-1}\left(\mathbb{R}^{n}\right)\right),
$$

is given by

$$
v_{P}(t, \cdot)=\mathbf{c}_{P}(t) f_{P}+\mathbf{s}_{P}(t) g_{P}+\int_{0}^{t} s_{P}(t-s) G_{P}(s, \cdot) d s .
$$

The same result holds if $P$ is exchanged with $S$, and both results hold for all $t_{0}>0$.

Proof. Following pages 816-818 of Smith (1998) we produce families of operators c $(t)$ and s $(t)$, respectively bounded and regularizing of degree 1 on $H^{s}\left(\mathbb{R}^{n}\right)$ for all $s$, with $\mathrm{c}(0)=I, \partial_{t} \mathrm{c}(0)=0, \mathrm{~s}(0)=0, \partial_{t} \mathrm{~s}(0)=I$, such that for $0 \leq s \leq 3$,

$$
\begin{aligned}
& \left(\partial_{t}^{2}-(2 \mu+\lambda) \Delta\right) \mathrm{c}(t)=T_{0}(t): H^{s}\left(\mathbb{R}^{n}\right) \rightarrow H^{s-1}\left(\mathbb{R}^{n}\right), \\
& \left(\partial_{t}^{2}-(2 \mu+\lambda) \Delta\right) \mathrm{s}(t)=T_{1}(t): H^{s-1}\left(\mathbb{R}^{n}\right) \rightarrow H^{s-1}\left(\mathbb{R}^{n}\right) .
\end{aligned}
$$

We set $\mathrm{c}_{P}(t)=\Pi_{P} \mathrm{c}(t) \Pi_{P}, \mathrm{~s}_{P}(t)=\Pi_{P} \mathrm{~s}(t) \Pi_{P}$, and observe that, for $0 \leq s \leq 2$,

$$
\begin{aligned}
& \left(\partial_{t}^{2}-A_{P P}\right) \mathrm{c}_{P}(t)=T_{P, 0}(t): H^{s}\left(\mathbb{R}^{n}\right) \rightarrow H^{s-1}\left(\mathbb{R}^{n}\right), \\
& \left(\partial_{t}^{2}-A_{P P}\right) \mathrm{s}_{P}(t)=T_{P, 1}(t): H^{s-1}\left(\mathbb{R}^{n}\right) \rightarrow H^{s-1}\left(\mathbb{R}^{n}\right) .
\end{aligned}
$$

To see this, we use the decomposition (2.11) to see that

$$
A_{P P} \mathrm{c}_{P}(t)-(2 \mu+\lambda) \Delta \mathrm{c}_{P}(t): H^{s}\left(\mathbb{R}^{n}\right) \rightarrow H^{s-1}\left(\mathbb{R}^{n}\right), \quad 0 \leq s \leq 2 .
$$


As in the discussion following (2.11), the commutator

$$
\left[\Pi_{P},(2 \mu+\lambda) \Delta\right]: H^{s}\left(\mathbb{R}^{n}\right) \rightarrow H^{s-1}\left(\mathbb{R}^{n}\right), \quad 0 \leq s \leq 2,
$$

and (3.2) follows from (3.1) and (2.11). The exact evolution operators $\mathbf{c}_{P}$ and $\mathbf{s}_{P}$ are obtained from $\mathbf{c}_{P}$ and $\mathrm{s}_{P}$ following pages 819-820 of Smith (1998), using a convergent iteration. For example,

$$
\mathbf{c}_{P}(t)=\mathrm{c}_{P}(t)+\int_{0}^{t} \mathrm{~s}_{P}(t-s) T(s) d s,
$$

where $T(t)$ is the solution to

$$
T(t)+\int_{0}^{t} T_{P, 1}(t-s) T(s) d s=-T_{P, 0}(t) .
$$

We observe here that, since $c_{P}(t), \mathrm{s}_{P}(t)$ and $T_{P, 0}(t)$ are orthogonal to $\Pi_{S}$ on both the right and left, it follows that so is $\mathbf{c}_{P}(t)$.

The bounds on $\partial_{t} \mathbf{c}_{P}(t)$, etc., follow similarly, using the fact that $\partial_{t} \mathrm{c}(t)$ and $\partial_{t} \mathrm{~s}(t)$ respectively map $H^{s}\left(\mathbb{R}^{n}\right) \rightarrow H^{s-1}\left(\mathbb{R}^{n}\right)$ and $H^{s-1}\left(\mathbb{R}^{n}\right) \rightarrow H^{s-1}\left(\mathbb{R}^{n}\right)$ for all $s$.

Suppose that $w$ is the solution to the linear elastic system (1.1). We pose

$$
v=(I+K) w, \quad v_{P}=\Pi_{P} v, \quad v_{S}=\Pi_{S} v .
$$

By (2.4) we can then write

$$
\begin{aligned}
\partial_{t}^{2} v_{P} & =\Pi_{P}(I+K) A(x, D) w \\
& =A_{P P}(I+K) w+R w \\
& =A_{P P} v_{P}+R(I+K)^{-1} v
\end{aligned}
$$

where at the last stage we use (2.10) to invert $(I+K)$ on the range of Sobolev spaces we will consider for $w$. Similar considerations apply to $v_{S}$, hence we have the system

$$
\left\{\begin{array}{lll}
\left(\partial_{t}^{2}-A_{P P}\right) v_{P}=R_{P} v, & \left.v_{P}\right|_{t=0}=\tilde{f}_{P}, & \left.\partial_{t} v_{P}\right|_{t=0}=\tilde{g}_{P}, \\
\left(\partial_{t}^{2}-A_{S S}\right) v_{S}=R_{S} v, & \left.v_{S}\right|_{t=0}=\tilde{f}_{S}, & \left.\partial_{t} v_{S}\right|_{t=0}=\tilde{g}_{S},
\end{array}\right.
$$

where $R_{P}$ and $R_{S}$ are bounded on $L^{2}\left(\mathbb{R}^{n}\right)$. Here, we have set $\tilde{f}_{P}=\Pi_{P}(I+K) f$ and $\tilde{g}_{P}=\Pi_{P}(I+K) g$, and similarly for $\tilde{f}_{S}, \tilde{g}_{S}$.

The system (3.3) is equivalent to the integral system,

$$
\left\{\begin{array}{l}
v_{P}(t)=\mathbf{c}_{P}(t) \tilde{f}_{P}+\mathbf{s}_{P}(t) \tilde{g}_{P}+\int_{0}^{t} \mathbf{s}_{P}(t-s) R_{P} v(s, \cdot) d s \\
v_{S}(t)=\mathbf{c}_{S}(t) \tilde{f}_{S}+\mathbf{s}_{S}(t) \tilde{g}_{S}+\int_{0}^{t} \mathbf{s}_{S}(t-s) R_{S} v(s, \cdot) d s
\end{array}\right.
$$

We now restrict attention to data $f \in H^{s}\left(\mathbb{R}^{n}\right), g \in H^{s-1}\left(\mathbb{R}^{n}\right)$, with $0 \leq s \leq 1$. Then

$$
\mathbf{c}_{P}(t) \tilde{f}_{P}+\mathbf{s}_{P}(t) \tilde{g}_{P} \in C\left(\left[-t_{0}, t_{0}\right], H^{s}\left(\mathbb{R}^{n}\right)\right),
$$

and the same holds with $P$ replaced by $S$. For $0 \leq s \leq 1$, then $s_{P}(t-s) R_{P}$ is a bounded map on $H^{s}\left(\mathbb{R}^{n}\right)$, and similarly with $P$ replaced by $S$, so the Volterra type system (3.4) can be solved by iteration to yield a solution

$$
v=v_{P}+v_{S} \in C\left(\left[-t_{0}, t_{0}\right], H^{s}\left(\mathbb{R}^{n}\right)\right) .
$$


The solution takes the form

$$
v=\mathbf{c}_{P}(t) \tilde{f}_{P}+\mathbf{s}_{P}(t) \tilde{g}_{P}+\mathbf{c}_{S}(t) \tilde{f}_{S}+\mathbf{s}_{S}(t) \tilde{g}_{S}+\mathbf{r}(t)(\tilde{f}, \tilde{g}),
$$

where $\mathbf{r}(t): L^{2}\left(\mathbb{R}^{n}\right) \oplus H^{-1}\left(\mathbb{R}^{n}\right) \rightarrow H^{1}\left(\mathbb{R}^{n}\right)$ and $\partial_{t} \mathbf{r}(t): L^{2}\left(\mathbb{R}^{n}\right) \oplus H^{-1}\left(\mathbb{R}^{n}\right) \rightarrow L^{2}\left(\mathbb{R}^{n}\right)$, with both $\mathbf{r}(t)$ and $\partial_{t} \mathbf{r}(t)$ strongly continuous in $t$.

We next write $\tilde{f}=(I+K) f, \tilde{g}=(I+K) g$, and $w=(I+K)^{-1} v$. By $(2.3)$,

$$
(I+K)^{-1}-I: H^{s}\left(\mathbb{R}^{n}\right) \rightarrow H^{s+1}\left(\mathbb{R}^{n}\right) \quad \text { for } \quad-1 \leq s \leq 1 .
$$

Consequently,

$$
(I+K)^{-1} \mathbf{c}_{P}(t)(I+K)-\mathbf{c}_{P}(t): L^{2}\left(\mathbb{R}^{n}\right) \rightarrow H^{1}\left(\mathbb{R}^{n}\right) .
$$

By similar results for the other terms, we have the following corollary (where $\mathbf{r}(t)$ is of a different form than above).

Theorem 3.2 Let $w(t, x)$ solve the Cauchy problem (1.1), with data $f \in L^{2}\left(\mathbb{R}^{n}\right), g \in H^{-1}\left(\mathbb{R}^{n}\right)$. Let $\left(f_{P}, g_{P}\right)=\Pi_{P}(f, g)$, and $\left(f_{S}, g_{S}\right)=\Pi_{S}(f, g)$. Then $w$ may be written as

$$
w(t, \cdot)=\mathbf{c}_{P}(t) f_{P}+\mathbf{s}_{P}(t) g_{P}+\mathbf{c}_{S}(t) f_{S}+\mathbf{s}_{S}(t) g_{S}+\mathbf{r}(t)(f, g),
$$

where $\mathbf{r}(t)$ is bounded from $L^{2}\left(\mathbb{R}^{n}\right) \oplus H^{-1}\left(\mathbb{R}^{n}\right)$ to $H^{1}\left(\mathbb{R}^{n}\right)$, and $\partial_{t} \mathbf{r}(t)$ is bounded from $L^{2}\left(\mathbb{R}^{n}\right) \oplus H^{-1}\left(\mathbb{R}^{n}\right)$ to $L^{2}\left(\mathbb{R}^{n}\right)$. Both $\mathbf{r}(t)$ and $\partial_{t} \mathbf{r}(t)$ are strongly continuous in $t$.

In particular, if the initial data for $w$ is a pure $P$ mode, in that $f_{S}=g_{S}=0$, then

$$
\Pi_{S} w=\Pi_{S} \mathbf{r}(t)(f, g) \in C\left(\left[-t_{0}, t_{0}\right], H^{1}\left(\mathbb{R}^{n}\right)\right),
$$

provided that $f_{P} \in L^{2}\left(\mathbb{R}^{n}\right)$ and $g_{P} \in H^{-1}\left(\mathbb{R}^{n}\right)$. This can be viewed as saying that the $P \rightarrow S$ coupling is regularizing of order 1 , and the same holds for pure $S$ modes.

This decoupling can also be expressed in terms of mapping properties of the components for the solution operator to (1.1). An immediate result of Theorem 3.2 is the following.

Corollary 3.3 Let the solution to (1.1) be written in the form

$$
w(t, \cdot)=\mathbf{c}(t) f+\mathbf{s}(t) g,
$$

and decompose $\mathbf{c}=\mathbf{c}_{P P}+\mathbf{c}_{P S}+\mathbf{c}_{S P}+\mathbf{c}_{S S}, \mathbf{s}=\mathbf{s}_{P P}+\mathbf{s}_{P S}+\mathbf{s}_{S P}+\mathbf{s}_{S S}$, where $\mathbf{c}_{P P}=\Pi_{P} \mathbf{c} \Pi_{P}$, etc. Then with $\mathbf{c}_{P}, \mathbf{c}_{S}, \mathbf{s}_{P}, \mathbf{s}_{S}$ as in Theorem 3.1, we have

$$
\begin{aligned}
\mathbf{c}_{P P}(t)-\mathbf{c}_{P}(t): L^{2}\left(\mathbb{R}^{n}\right) \rightarrow H^{1}\left(\mathbb{R}^{n}\right), \\
\mathbf{c}_{P S}(t): L^{2}\left(\mathbb{R}^{n}\right) \rightarrow H^{1}\left(\mathbb{R}^{n}\right), \\
\mathbf{s}_{P P}(t)-\mathbf{s}_{P}(t): H^{-1}\left(\mathbb{R}^{n}\right) \rightarrow H^{1}\left(\mathbb{R}^{n}\right), \\
\mathbf{s}_{P S}(t): H^{-1}\left(\mathbb{R}^{n}\right) \rightarrow H^{1}\left(\mathbb{R}^{n}\right) .
\end{aligned}
$$

All results hold with $P$ and $S$ interchanged, and are strongly continuous in $t$.

Futhermore, in each case the time derivative of the operator is a bounded map from the indicated domain into $L^{2}\left(\mathbb{R}^{n}\right)$. For example,

$$
\partial_{t} \mathbf{c}_{P P}(t)-\partial_{t} \mathbf{c}_{P}(t): L^{2}\left(\mathbb{R}^{n}\right) \rightarrow L^{2}\left(\mathbb{R}^{n}\right),
$$

with strongly continuous dependence on $t$. 
The corollary follows from Theorem 3.2 since all operators listed arise as compositions of $\mathbf{r}(t)$ with the projections $\Pi_{P}$ and $\Pi_{S}$.

We remark that if the stronger regularity assumption is made, $\mu, \lambda \in C^{k, 1}$ with $k \geq 2$, then the range of Sobolev indexes $s$ on which $\mathbf{r}(t)$ is regularizing of order 1 increases, and Corollary 3.3 thus applies to a range of $H^{s}\left(\mathbb{R}^{n}\right)$. Alternatively, by adding successive terms to $K$, the system (3.3) for $v=(I+K) w$ can be decoupled to higher order, that is, with $R_{P}$ and $R_{S}$ of negative order; see Taylor (1975) for the case of smooth $\mu, \lambda$ where $R_{P}$ and $R_{S}$ are smoothing operators. This requires adding lower order terms to $A_{P P}$ and $A_{S S}$. The order of gain in regularity in Corollary 3.3 cannot be increased, however, since it is limited by the term of order -1 in $K$.

\section{Acknowledgments}

The research of Brytik, de Hoop, and Uhlmann was supported in part under NSF CMG grant EAR0724644; Uhlmann was also partly supported by a Walker Family Endowed Professorship. The research of Smith was supported under NSF grant DMS-0654415.

\section{References}

Calderón, A. (1965). Commutators of singular integral operators. Proc. Nat. Acad. Sci. U.S.A. 53:10921099.

Dencker, N. (1982). On the propagation of polarization sets for systems of real principal type. J. Functional Analysis 46:351-372.

Langston, C.A. (1979). Structure under Mount Rainier, Washington, inferred from teleseismic body waves. J. Geophys. Res. 84:4749-4762.

Lin, C.L., Nakamura, G., Uhlmann, G., Wang, J.N. (2010). Quantitative unique continuation for the Lamé system with less regular coefficients. Preprint, arXiv:1005.3382v1.

Smith, H.F. (1998). A parametrix construction for wave equations with $C^{1,1}$ coefficients. Ann. Inst. Fourier, Grenoble 4:797-835.

Stolk, C. (2000). Microlocal analysis of a seismic linearized inverse problem. Wave Motion 32:267-290.

Stolk, C., de Hoop, M.V. (2002). Microlocal analysis of seismic inverse scattering in anisotropic elastic media. Comm. Pure Appl. Math. 55:261-301.

Stolk, C., de Hoop, M.V. (2006). Seismic inverse scattering in the downward continuation approach. Wave Motion 43:579-598.

Taylor, M. (1975). Reflection of singularities of solutions to systems of differential equations. Comm. Pure Appl. Math. 28:457-478.

Wang, J.N. (1998). On the relation between singularities of coefficients and singularities of reflected waves in the Lamé system. Inverse Problems 14:733-743.

Vinnik, L. (1977). Detection of waves converted from P to SV in the mantle. Phys. Earth Planet. Inter. 15:39-45. 